PROCEEDINGS OF THE

AMERICAN MATHEMATICAL SOCIETY

Volume 128, Number 6, Pages 1833-1838

S 0002-9939(99)05207-7

Article electronically published on September 30, 1999

\title{
AN OBSTRUCTION TO THE CONFORMAL COMPACTIFICATION OF RIEMANNIAN MANIFOLDS
}

\author{
SEONGTAG KIM
}

(Communicated by Christopher Croke)

\begin{abstract}
In this paper, we study noncompact complete Riemannian $n$ manifolds with $n \geq 3$ which are not pointwise conformal to subdomains of any compact Riemannian $n$-manifold. For this, we compare the Sobolev Quotient at infinity of a noncompact complete Riemannian manifold with that of the singular set in a compact Riemannian manifold using the method for the Yamabe problem.
\end{abstract}

\section{Introduction}

In this paper, we study noncompact complete Riemannian $n$-manifolds with $n \geq 3$ which are not pointwise conformal to subdomains of any compact Riemannian $n$-manifold. We show that the conformal structure at infinity provides an obstruction to the conformal compactification of certain noncompact complete Riemannian manifolds. This obstruction is interesting because Nomizu and Ozeki [6] showed that any Riemannian manifold admits a complete metric by the conformal change of metric.

First, we calculate the Sobolev Quotient of a singular set in a given compact Riemannian manifold using the method which was developed for the Yamabe problem. Second, we compare the Sobolev Quotient at infinity of a given noncompact complete Riemannian manifold with the Sobolev Quotient of a singular set in a compact Riemannian manifold. To do this we introduce some notation:

$$
Q(M, g) \equiv \inf _{u \in C_{0}^{\infty}(M)} \frac{\int_{M}|\nabla u|^{2}+C_{n} S u^{2} d V_{g}}{\left(\int_{M} u^{2 n /(n-2)} d V_{g}\right)^{(n-2) / n}}
$$

and

$$
\overline{Q(M, g)} \equiv \lim _{r \rightarrow \infty} \inf _{u \in C_{0}^{\infty}\left(M-B_{r}\right)} \frac{\int_{M-B_{r}}|\nabla u|^{2}+C_{n} S u^{2} d V_{g}}{\left(\int_{M-B_{r}} u^{2 n /(n-2)} d V_{g}\right)^{(n-2) / n}},
$$

Received by the editors July 22, 1998.

1991 Mathematics Subject Classification. Primary 53C21; Secondary 58 G30.

Key words and phrases. Scalar curvature, Yamabe problem, conformal metric.

The author was supported in part by KOSEF96070102013 and BSRI 97-1419 Ministry of Education. 
where $C_{n}=\frac{n-2}{4(n-1)}, S$ is the scalar curvature of $(M, g)$ and $B_{r}$ is the ball of radius $r$ with center at a fixed point $x_{0}$.

We also use the notation $Q(M)$ for $Q(M, g)$ and $Q\left(S^{n}\right)$ for $Q\left(S^{n}, g_{0}\right)$, where $g_{0}$ is the standard metric. Note that $\overline{Q(M, g)}$ is well defined since

$$
\begin{aligned}
& \inf _{u \in C_{0}^{\infty}\left(M-B_{r}\right)} \frac{\int_{M-B_{r}}|\nabla u|^{2}+C_{n} S u^{2} d V_{g}}{\left(\int_{M-B_{r}} u^{2 n /(n-2)} d V_{g}\right)^{(n-2) / n}} \\
& \leq \inf _{u \in C_{0}^{\infty}\left(M-B_{r+1}\right)} \frac{\int_{M-B_{r+1}}|\nabla u|^{2}+C_{n} S u^{2} d V_{g}}{\left(\int_{M-B_{r+1}} u^{2 n /(n-2)} d V_{g}\right)^{(n-2) / n}} .
\end{aligned}
$$

$\overline{Q(M, g)}$ was used for a study of the Yamabe problem on noncompact complete Riemannian manifolds by Kim [4]. Obstructions for the existence of a complete conformal metric with prescribed scalar curvature on a subdomain $K-\Gamma$ of a compact Riemannian manifold $(K, h)$ were studied by Aviles and McOwen [2, Schoen and Yau [8], Delanoe [3] and McOwen [5] using the dimension of $\Gamma$. In this paper, we have an obstruction for the conformal compactification of a noncompact complete Riemannian manifold using the conformal structure at infinity.

\section{The Sobolev Quotient of a singular set}

In this section, we show that the Sobolev Quotient of a singular set is the same as that of the standard sphere. Let us denote the $n$ dimensional Riemannian volume of a subset $\Omega$ of $(M, g)$ by $|\Omega|$. We state our theorem.

Theorem 2.1. Let $(M, g)$ be a Riemannian manifold of dimension $n \geq 3$. Assume that there exists a sequence $\left\{\Gamma_{i}\right\}$ of smooth bounded open subsets of $(M, g)$ with $\left|\Gamma_{i}\right| \rightarrow 0$ and $\Gamma_{i} \subset \Gamma_{1}$ for $i=1,2, \cdots$. Then we have $\lim _{i \rightarrow \infty} Q\left(\Gamma_{i}\right)=Q\left(S^{n}\right)$.

Proof. Assume that there exists a subsequence $\left\{\Gamma_{i_{k}}\right\}$ of $\left\{\Gamma_{i}\right\}$ such that

$$
\lim _{i \rightarrow \infty} Q\left(\Gamma_{i_{k}}\right)<Q\left(S^{n}\right)
$$

We use the same notation $\left\{\Gamma_{i}\right\}$ for this subsequence. By the work on the Yamabe problem in the compact case (see [9], [1], [7], and [8]), there exists a positive solution $u_{i} \in C^{\infty}\left(\Gamma_{i}\right)$ with

$$
\begin{aligned}
& -\Delta u_{i}+C_{n} S u_{i}=q_{i} u_{i}^{(n+2) /(n-2)} \text { on } \Gamma_{i}, \\
& \int_{\Gamma_{i}} u_{i}^{2 n /(n-2)} d V_{g}=1 \text { and } u_{i}=0 \text { on } \partial \Gamma_{i},
\end{aligned}
$$

where $q_{i}=Q\left(\Gamma_{i}\right)$. We extend the domain of $u_{i}$ by defining $u_{i}=0$ on the outside of $\Gamma_{i}$. By multiplying (2.2) by $u_{i}^{1+2 b}$ where $b>0$ and integrating over $\Gamma_{i}$,

$$
q_{i} \int_{\Gamma_{i}} u_{i}^{2 n /(n-2)+2 b} d V_{g}=\int_{\Gamma_{i}} \frac{1+2 b}{(1+b)^{2}}\left|\nabla u_{i}^{1+b}\right|^{2}+C_{n} S u_{i}^{2+2 b} d V_{g} .
$$


Using Hölder's inequality,

$$
\begin{aligned}
& \int_{\Gamma_{i}} u_{i}^{2 n /(n-2)+2 b} d V_{g} \\
& \left.\leq \underset{\Gamma_{\Gamma_{i}}}{(1+b) 2 n /(n-2)} d V_{g}\right)^{\frac{n-2}{n}}\left(\int_{\Gamma_{i}} u_{i}^{(2 n /(n-2)+2 b-(1+b) 2) \frac{n}{2}} d V_{g}\right)^{\frac{2}{n}} \\
& \left.\leq \underset{\Gamma_{\Gamma_{i}}}{(1+b) 2 n /(n-2)} d V_{g}\right)^{\frac{n-2}{n}} .
\end{aligned}
$$

By the Sobolev Embedding Theorem on Riemannian manifolds for $u_{i}$ on $\Gamma_{i}, \Gamma_{i} \subset$ $\Gamma_{1} \subset M$ (note $\overline{\Gamma_{1}}$ is compact), and (2.3), for any given $\epsilon>0$, there exists $C(\epsilon)$ with

$$
\begin{aligned}
& \left(\int_{\Gamma_{i}} u_{i}^{(1+b) 2 n /(n-2)} d V_{g}\right)^{\frac{n-2}{n}} \\
& \leq(1+\epsilon) \frac{1}{Q\left(S^{n}\right)} \int_{\Gamma_{i}}\left|\nabla u_{i}^{1+b}\right|^{2} d V_{g}+C(\epsilon) \int_{\Gamma_{i}} u_{i}^{2+2 b} d V_{g} \\
& \leq(1+\epsilon) \frac{1}{Q\left(S^{n}\right)} \frac{(1+b)^{2}}{(1+2 b)}\left(\int_{\Gamma_{i}} q_{i} u_{i}^{2 n /(n-2)+2 b}-C_{n} \int_{\Gamma_{i}} S u_{i}^{2+2 b} d V_{g}\right) \\
& \quad+C(\epsilon) \int_{\Gamma_{i}} u_{i}^{2+2 b} d V_{g} .
\end{aligned}
$$

From (2.4),

$$
\begin{aligned}
& \left(\int_{\Gamma_{i}} u_{i}^{(1+b) 2 n /(n-2)} d V_{g}\right)^{\frac{n-2}{n}} \\
& \leq(1+\epsilon) \frac{1}{Q\left(S^{n}\right)} \frac{(1+b)^{2}}{(1+2 b)}\left(q_{i}\left(\int_{\Gamma_{i}} u_{i}^{(1+b) 2 n /(n-2)} d V_{g}\right)^{\frac{n-2}{n}}\right. \\
& \left.-C_{n} \int_{\Gamma_{i}} S u_{i}^{2+2 b} d V_{g}\right)+C(\epsilon) \int_{\Gamma_{i}} u_{i}^{2+2 b} d V_{g} .
\end{aligned}
$$

Since $q_{i}<c<Q\left(S^{n}\right)$ for some $c$, we can take $\epsilon>0$ and $0<b<\frac{2}{n-2}$ so that

$$
(1+\epsilon) \frac{q_{i}}{Q\left(S^{n}\right)} \frac{(1+b)^{2}}{(1+2 b)}<c_{0}<1
$$

for some constant $c_{0}$. Therefore, we have

$$
\begin{aligned}
& \left(1-(1+\epsilon) \frac{q_{i}}{Q\left(S^{n}\right)} \frac{(1+b)^{2}}{(1+2 b)}\right)\left(\int_{\Gamma_{i}} u_{i}^{(1+b) 2 n /(n-2)} d V_{g}\right)^{\frac{n-2}{n}} \\
& +C \int_{\Gamma_{i}} S u_{i}^{2+2 b} d V_{g} \\
& \leq C(\epsilon) \int_{\Gamma_{i}} u_{i}^{2+2 b} d V_{g}
\end{aligned}
$$


where $C$ is a general positive constant independent of $i$. We may assume that $\left|\Gamma_{i}\right|<1$. Using Hölder's inequality,

$$
\begin{aligned}
\int_{\Gamma_{i}} u_{i}^{2+2 b} d V_{g} & \leq\left(\int_{\Gamma_{i}} u_{i}^{2 n /(n-2)} d V_{g}\right)^{t_{1}}\left|\Gamma_{i}\right|^{1-t_{1}} \\
& \leq\left|\Gamma_{i}\right|^{1-t_{1}}<1,
\end{aligned}
$$

where $t_{1}=\frac{(1+b)(n-2)}{n}$.

Finally, we have

$$
\left(\int_{M} u_{i}^{(1+b) 2 n /(n-2)} d V_{g}\right)^{\frac{n-2}{n}}=\left(\int_{\Gamma_{i}} u_{i}^{(1+b) 2 n /(n-2)} d V_{g}\right)^{\frac{n-2}{n}} \leq A_{1},
$$

where $A_{1}$ does not depend on $i$.

From (2.9), we have

$$
\begin{aligned}
1=\int_{\Gamma_{i}} u_{i}^{2 n /(n-2)} d V_{g} & \leq\left(\int_{\Gamma_{i}} u_{i}^{(1+b) 2 n /(n-2)} d V_{g}\right)^{t}\left|\Gamma_{i}\right|^{s} \\
& \leq A_{1}^{n t /(n-2)}\left|\Gamma_{i}\right|^{s},
\end{aligned}
$$

where $t=\frac{1}{1+b}$ and $s=\frac{b}{1+b}$.

Since $\left|\Gamma_{i}\right| \rightarrow 0$, (2.10) does not hold. This contradiction comes from (2.1). Thus Theorem 2.1 is proved.

\section{Applications on noncompact COMPlete Riemannian manifolds}

Using Theorem 2.1. we show that there exists an obstruction to the conformal compactification of certain noncompact complete Riemannian manifolds.

Theorem 3.1. Let $(M, g)$ be a noncompact complete Riemannian manifold of dimension $n \geq 3$ with $\overline{Q(M, g)}<Q\left(S^{n}\right)$. Then $(M, g)$ is not pointwise conformal to a subdomain of any compact Riemannian n-manifold.

Proof. Assume that $(M, g)$ is pointwise conformal to a subdomain $\left(M, u^{4 /(n-2)} g\right)$ in $(K, h)$, where $(K, h)$ is a compact Riemannian $n$-manifold. Take smooth compact domain $X_{i}$ in $M$ with $X_{i} \subset \overline{X_{i}} \subset X_{i+1}$ so that $\operatorname{Vol}\left(M-X_{i}, u^{4 /(n-2)} g\right) \rightarrow 0$ as $i \rightarrow \infty$, where $\operatorname{Vol}\left(M-X_{i}, u^{4 /(n-2)} g\right)$ is the volume of $M-X_{i}$ with the metric $u^{4 /(n-2)} g$. We let $|\cdot|_{h}\left(|\cdot|_{g}\right)$ be the length of the tangent vector and $S_{h}\left(S_{g}\right)$ be the scalar curvature with the metric $h$ (respectively $g$ ).

Since we assume that $\overline{Q(M, g)}<Q\left(S^{n}\right)$, there exists a smooth function $f_{i} \in$ $C_{0}^{\infty}\left(M-\overline{X_{i}}\right)$ with

$$
\frac{\int_{M-\overline{X_{i}}}\left|\nabla f_{i}\right|_{g}^{2}+C_{n} S_{g} f_{i}^{2} d V_{g}}{\left(\int_{M-\overline{X_{i}}} f_{i}^{2 n /(n-2)} d V_{g}\right)^{(n-2) / n}}<Q\left(S^{n}\right)-c_{0}
$$

for a positive constant $c_{0}$ and $i=1,2, \cdots$.

We take a smooth bounded open subset $Y_{i}$ of $M$ with $X_{i} \subset \overline{X_{i}} \subset Y_{i}$ and

$$
Z_{i} \subset Y_{i}-X_{i} \subset M-X_{i}
$$


where $Z_{i}$ is the support of $f_{i}$. By the conformal invariance of $Q\left(Y_{i}-\overline{X_{i}}, h\right)$,

$$
\begin{aligned}
Q\left(Y_{i}-\overline{X_{i}}, h\right) & =Q\left(Y_{i}-\overline{X_{i}}, u^{4 /(n-2)} g\right) \\
& \leq \frac{\int_{\bar{i}}-\overline{X_{i}}}{\left(\int_{Y_{i}-\overline{X_{i}}}\left(f_{i} / u\right)^{2 n /(n-2)} d V_{h}\right)^{(n-2) / n}} \\
& \leq \frac{\int_{Y_{i}-\overline{X_{i}}}\left|\nabla f_{i}\right|_{g}^{2}+C_{n} S_{g} f_{i}^{2} d V_{g}}{\left(\int_{Y_{i}-\overline{X_{i}}} f_{i}^{2 n /(n-2)} d V_{g}\right)^{(n-2) / n}} \\
& \leq Q\left(S^{n}\right)-c_{0} .
\end{aligned}
$$

Since the $\operatorname{Vol}\left(Y_{i}-\overline{X_{i}}, u^{4 /(n-2)} g\right) \rightarrow 0$, (3.1) contradicts Theorem 2.1. Theorem 3.1 is proved.

Using Theorem 3.1, we have the following.

Corollary 3.2. Let $(M, g)$ be a noncompact complete Riemannian manifold of dimension $n \geq 3$. Assume that there exists a positive number $c_{0}$ and a sequence $\left\{\Omega_{i}\right\}$ of smooth open sets where $\Omega_{i} \subset M-B_{i}\left(x_{0}\right)$ for $i=1,2, \cdots$ and $Q\left(\Omega_{i}\right)<Q\left(S^{n}\right)-c_{0}$. Then $(M, g)$ is not pointwise conformal to a subdomain of any compact Riemannian $n$-manifold.

Example 3.3. Let $B(p, r)$ be the Euclidean ball of radius $r$ with center $p$ in $\left(R^{n}, \delta_{i j}\right)$ with $n \geq 3$. Take any smooth metric $h$ on $B(p, 2)$ so that $h=\delta_{i j}$ on $B(p, 2)-B(p, 1)$ and $h$ is not conformally flat at the center $p$. Then $Q(B(p, 2), h)<$ $Q\left(S^{n}\right)$ by the resolution of the Yamabe problem. Consider $\left(R^{n}, g\right)$ with $n \geq 3$, where $g=h$ on each Euclidean balls $B((5 n, 0), 2)$ with center $(5 n, 0) \in R \times R^{n-1}$ and radius 2 in the Euclidean norm, for $n=1,2,3, \cdots$ and $g=\delta_{i j}$ on $R^{n}-$ $\bigcup B((5 n, 0), 2)$. Then $\left(R^{n}, g\right)$ is not pointwise conformal to a subdomain of any compact Riemannian $n$-manifold, since $\overline{Q\left(R^{n}, g\right)}<Q\left(S^{n}\right)$.

\section{ACKNOWLEDGEMENTS}

The author thanks Patricio Aviles for his encouragement on this work.

\section{REFERENCES}

1. T. Aubin, Equations différentielles non linéaires et problème de Yamabe concernant la courbure scalaire, J. Math. Pures Appl. n 55 (1976) 269-296. MR 55:4288

2. P. Aviles and R. McOwen, Complete conformal metrics with negative scalar curvature in compact Riemannian manifolds, Duke Math. J. 56 (1988) 225-239. MR 89b:58224

3. P. Delanoe, Generalized stereographic projections with prescribed scalar curvature, Geometry and nonlinear partial differential equations, (Contemp. Math., vol 127 pp. 17-25) Providence, A.M.S. 1992 [MR 93e:53045

4. S. Kim, Scalar curvature on noncompact complete Riemannian manifolds, Nonlinear Analysis 26 (1996) 1985-1993. MR 97a:53056

5. R. McOwen, Singularities and the conformal scalar curvature equation, Geometry and nonlinear partial differential equations (Contemp. Math., vol 127 pp. 221-233) Providence, A.M.S. 1992 MR 94b:53076

6. K. Nomizu and H. Ozeki, The existence of complete Riemannian metrics, Proc. Amer. Math. Soc. 12 (1961) 889-891. MR 24:A3610]

7. R. Schoen, Conformal deformation of a Riemannian metric to constant scalar curvature, J. Diff. Geom. 20 (1984) 479-495. MR 86i:58137 
8. R. Schoen and S. Yau, Conformally flat manifolds, Kleinian groups and scalar curvature, Invent. Math. 92 (1988) 47-71. MR 89c:58139

9. H. Yamabe, On a deformation of Riemannian structures on compact manifolds, Osaka Math. J. 12 (1960) 21-37. MR 23:A2847

Department of Mathematics, Sungkyunkwan University, Suwon 440-746, South Korea

E-mail address: stkim@yurim.skku.ac.kr 